\title{
Cryo-EM of Li Metal Battery Aging and Failure Mechanisms
}

Katherine Jungjohann ${ }^{1}$, Daniel Long ${ }^{1}$, Renae Gannon ${ }^{2}$, Steven Randolph ${ }^{3}$, Subrahmanyam Goriparti ${ }^{1}$, Laura Merrill $^{1}$ and Katharine Harrison ${ }^{1}$

${ }^{1}$ Sandia National Laboratories, Albuquerque, New Mexico, United States, ${ }^{2}$ University of Oregon, Eugene, Oregon, United States, ${ }^{3}$ Thermo Fisher Scientific, Hillsboro, Oregon, United States

Lithium metal is a challenging material to characterize, as it is low in atomic weight, air/water sensitive, and very electron beam sensitive. Although challenging, the technological need for its characterization is set by the increasing need for advanced energy storage technologies. Lithium metal anodes for rechargeable energy storage present a 50\% increase in energy density beyond commercial Li-ion batteries, though the cycling conditions needed for electric vehicles, for example, produce Li metal plating and stripping morphologies that are unsafe, unstable, and reduce the battery life. Understanding the source of these failure mechanisms, as well as the root interactions between the Li metal and the electrolytes are critical to providing engineering strategies for overcoming these commercialization barriers for the realization of Li metal anodes in rechargeable energy storage.

In the past several years, various groups have developed new cryogenic electron microscopy strategies for the characterization of Li metal anodes [1], and intact electrode/electrolyte interfaces [2]. The se tools range from sample preparation including cryo-focused ion beam (FIB), cryogenic transfer equipment [3], and cryogenic holders for scanning/transmission electron microscopy (S/TEM). Our work builds on these techniques for understanding the aging and failure mechanisms of Li metal anodes, by starting with cryogenic-TriBeam imaging of whole coin cell batteries frozen on a cryo-stage, for analysis of the intact component layers in crosssection. The athermal ultrashort pulse laser of the TriBeam can mill through material at rates over 10,000x those of standard FIB milling [4], which is exceptional for accessing representative cross-sections of the frozen battery stack. Using this method, we can put more detailed information from the cryo-FIB and cryo-S/TEM into perspective. Additionally, the TriBeam can localize interfaces of the sample that are of the greatest interest for informing a mechanistic understanding of the solid-liquid interfacial evolution that occurs with aging and battery cycling.

Our early data has identified that a main failure mechanism of Li metal anode batteries is caused by degradation of the separator polymer after over 100 lithium plating/stripping cycles. This failure mechanism has been completely overlooked with standard characterization mechanisms, as generally the separator is removed and discarded for access to the electrode surface for characterization. Images of the intact battery stack reveal that after 10's of cycles, the electrode/separator interface becomes intertwined, meaning that part of the electrode will remain with the separator when these two components are separated. Additionally, cryo-TriBeam analysis of the intact battery stack has confirmed that the quantity of solid-electrolyte-interphase products that form over many cycles is much more than anticipated [2], seen in Figure 1, leading to the consumption of much of the Li metal that was being transported between opposing electrodes. This understanding of the overarching processes occurring in the Li metal batteries will direct our future characterization with cryo-FIB, cryoscanning electron microscopy for energy dispersive $\mathrm{x}$-ray spectroscopy and electron backscatter diffraction, and cryo-S/TEM of the electrode/electrolyte interfaces [5]. 


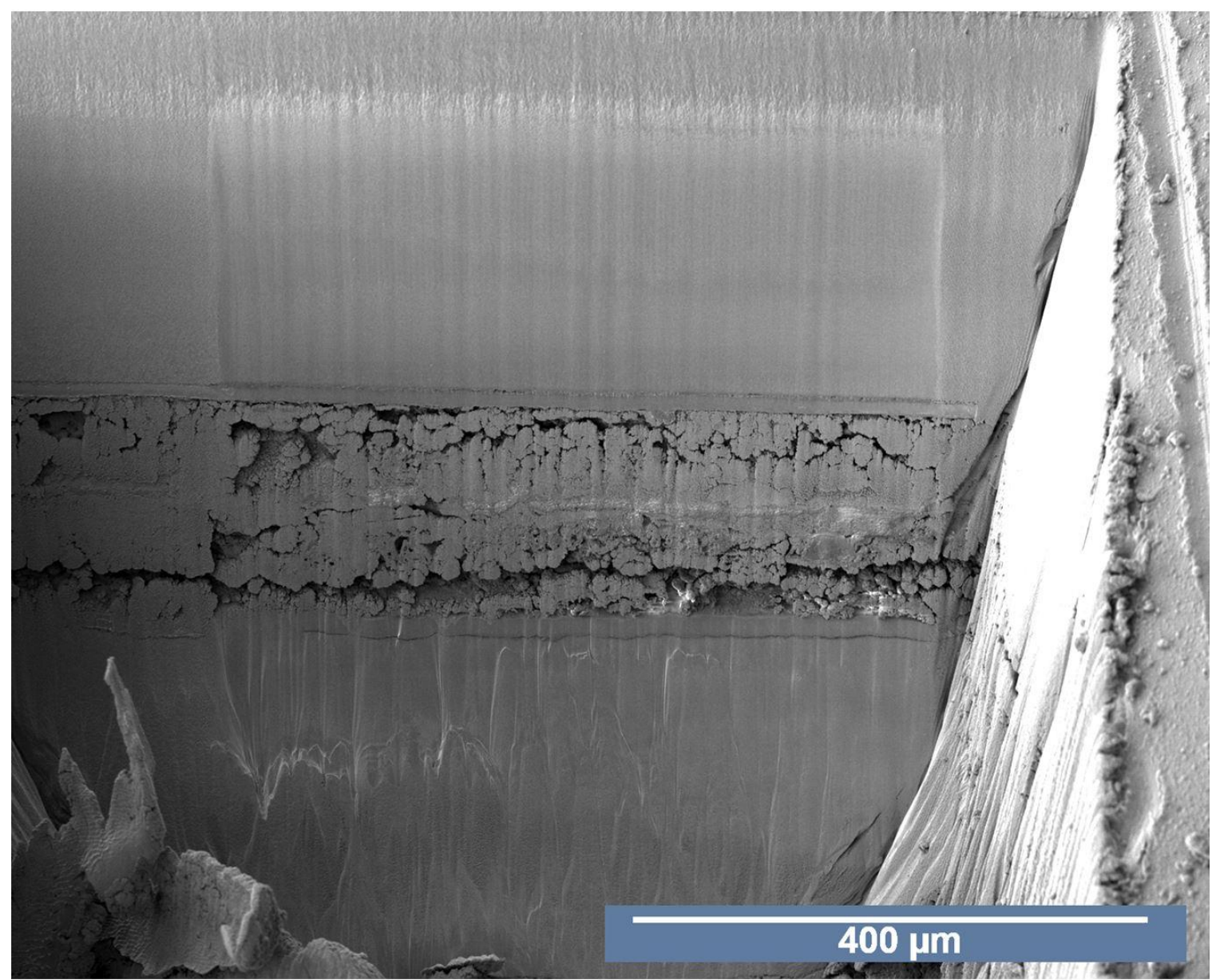

Figure 1. Scanning electron micrograph after cryo-TriBeam sectioning of an intact coin cell battery stack of a Li metal plated vs. a Li metal counter electrode in $4 \mathrm{M}$ lithium bis(fluorosulfulfonyl)imide in dimethoxyethane with a Celgard 2400 separator. Large crevices, in black, show the solid electrolyte interphase evolution that prevents a pathway for $\mathrm{Li}$ ions between the opposing electrodes in the coin cell.

\section{References}

[1] Y Li et al., Science 358 (2017), p. 506.

[2] MJ Zachman et al., Nature 560 (2018), p. 345.

[3] J He et al., Journal of Structural Biology 199 (2017), p. 114.

[4] MP Echlin et al., Materials Characterization 100 (2015), p. 1.

[5] This work was funded by Sandia National Laboratories' Laboratory Directed Research and Development program. It was performed, in part, at the Center for Integrated Nanotechnologies, an Office of Science User Facility operated for the U.S. Department of Energy (DOE) Office of Science. Sandia National Laboratories is a multi-mission laboratory managed and operated by National Technology and Engineering Solutions of Sandia, LLC., a wholly owned subsidiary of Honeywell International, Inc., for the U.S. DOE's National Nuclear Security Administration under contract DE-NA-0003525. The views expressed in the article do not necessarily represent the views of the U.S. DOE or the United States Government. 